\title{
Sporadic long-term variability in radio activity from a brown dwarf
}

\author{
A. Antonova ${ }^{1}$, J. G. Doyle ${ }^{1}$, G. Hallinan ${ }^{2}$, A. Golden ${ }^{2}$, and C. Koen ${ }^{3}$ \\ 1 Armagh Observatory, College Hill, Armagh BT61 9DG, N. Ireland \\ e-mail: tan@arm.ac.uk \\ 2 Computational Astrophysics Laboratory, I.T. Building, National University of Ireland, Galway, Ireland \\ 3 Department of Statistics, University of the Western Cape, Private Bag X17, Bellville, 7535 Cape, South Africa
}

Received 2 February 2007 / Accepted 11 June 2007

\section{ABSTRACT}

\begin{abstract}
Context. Radio activity has been observed in a large variety of stellar objects, including in the last few years, ultra-cool dwarfs. Aims. To explore the extent of long-term radio activity in ultra-cool dwarfs.

Methods. We use data taken over an extended period of $9 \mathrm{hr}$ from the Very Large Array of the source 2MASS J05233822-1403022 in September 2006, plus data taken in 2004.

Results. The observation taken in September 2006 failed to detect any radio activity at $8.46 \mathrm{GHz}$. A closer inspection of earlier data reveals that the source varied from a null detection on 3 May 2004, to $\approx 95 \mu \mathrm{Jy}$ on 17 May 2004, to $230 \mu \mathrm{Jy}$ on $18 \mathrm{June} 2004$. The lack of detection in September 2006 suggests at least a factor of ten flux variability at $8.46 \mathrm{GHz}$. Three short photometric runs did not reveal any optical variability.

Conclusions. In addition to the observed pulsing nature of the radio flux from another ultra-cool source, the present observations suggests that ultra-cool dwarfs may not just be pulsing but can also display long-term sporadic variability in their levels of quiescent radio emission. The lack of optical photometric variability suggests an absence of large-scale spots at the time of the latest VLA observations, although small very high latitude spots combined with a low inclination could cause very low amplitude rotational modulation which may not be measurable. We discuss this large variability in the radio emission within the context of both gyrosynchrotron emission and the electron-cyclotron maser, favoring the latter mechanism.
\end{abstract}

Key words. stars: activity - stars: atmospheres - stars: low-mass, brown dwarfs - radio continuum: stars - masers radiation mechanisms: general

\section{Introduction}

For ultra-cool stars and brown dwarfs, both $\mathrm{H} \alpha$ and X-ray emission decline in strength so that few field objects later than L5 exhibit evidence of activity at these frequencies (Schmidt et al. 2007, and references therein). This reduction in both chromospheric and coronal emission is in broad agreement with some theoretical models, i.e. the cool, dense atmospheres implying low ionization fractions and thus high electrical resistivities, leading to a decoupling of magnetic lines from the upper atmosphere (Mohanty et al. 2002).

Given the above considerations, it was generally assumed that radio emission from cool dwarfs and brown dwarfs would be either weak or absent. This was supported by the Güdel \& Benz (1993) empirical correlation between radio and X-ray emission which holds over many orders of magnitude. However, the detection by Berger et al. (2001) of radio emission from the M9 field brown dwarf LP 944-20 violated the above relation by four orders of magnitude. Further observations produced more radio active ultra-cool dwarfs (Berger 2002; Burgasser \& Putman 2005; Berger 2006; Phan-Bao et al. 2007). In Fig. 1 we plot the ratio of the radio to bolometric luminosity for all known radio active ultra-cool dwarfs compared to a selection of earlyto-mid M dwarfs taken from the literature. Until recently, the detected radio emission has been interpreted as incoherent gyrosynchrotron radiation from a population of non-thermal electrons (Berger 2002; Burgasser \& Putman 2005; Berger 2006; Osten et al. 2006).

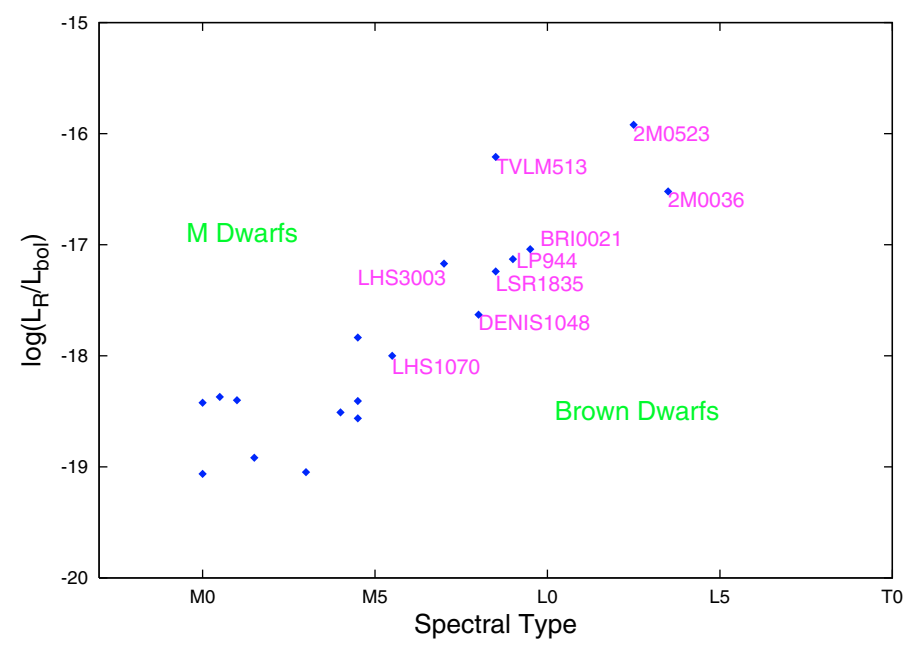

Fig. 1. Ratio of the radio to bolometric luminosity for all known radio active ultra-cool dwarfs, compared to a selection of early-to-mid M dwarfs. The present 2006 radio data for 2 M0523 is at least a factor of ten lower.

Berger et al. (2005) conducted simultaneous $4.9 \mathrm{GHz}$ and $8.5 \mathrm{GHz}$ observations of the L3.5 brown dwarf 2MASS J00361617+1821104 which yielded highly polarized emission in both bands. Furthermore, the emission was both periodic and variable, with the average flux and significance of the variability higher at $4.9 \mathrm{GHz}$. Due to the low net circular polarization 
and the broadband persistent nature of the emission, Berger et al. attributed it to the gyrosynchrotron process. Hallinan et al. (2006) detected periodic emission from the M9 dwarf TVLM 513-46546 with the similar properties to those detected from 2MASS J00361617+1821104 but, using geometrical constraints, suggested an alternative process, the electron cyclotron maser instability as the source of the dominant radio emission. In subsequent observations of the same dwarf, Hallinan et al. (2007) reported on $100 \%$ circularly polarized bursts with a periodicity that was in very good agreement with the rotational period of the dwarf, as confirmed by $I$-band photometric observations. From the duration of the bursts they constrained the size of the emitting source region to be less than 0.22 times the radius of the dwarf, estimating the brightness temperature of the radio emission. Those bursts conclusively confirmed that at least part of the emission was due to the electron cyclotron maser instability.

Considering the similarities in the properties of the radio emission of these two dwarfs, it is possible that the same mechanism is responsible for the emission in both cases. The above observations suggest the probable existence of large-scale, stable magnetic fields on at least some ultra-cool dwarfs. In this Letter we report on high sensitivity VLA observations of the radio active L 2.5 brown dwarf 2MASS J05233822-1403022, undertaken to investigate the production mechanism of the radio emission, as well as to search for rotational modulation of the emission.

\section{Observations and data reduction}

\subsection{Radio}

The observations of 2MASS J05233822-1403022 (hereafter 2M0523) were conducted with the NRAO Very Large Array $(\mathrm{VLA})^{1}$ for a duration of $\approx 9 \mathrm{~h}$ as part of a monitoring program on several previously detected ultra-cool dwarfs. 2M0523 was observed on 2006 September 23, at $8.45 \mathrm{GHz}(3.6 \mathrm{~cm})$. During the observations the array consisted of 23 antennas in the B configuration. We used the standard continuum mode with $2 \times 50$ contiguous bands, sampling every $10 \mathrm{~s}$. The flux density calibrator was 3 C 138, while the phase calibrator was 0513-219 with the time on source in a single scan being $12 \mathrm{~min}$, before moving to the phase calibrator for $2 \mathrm{~min}$.

For comparison, we obtained a set of observations of the same source from the public archive of the NRAO Data Archive System. These observations were conducted on 2004 May 3, May 17 and June 18, at $8.45 \mathrm{GHz}$, each for a duration of $\sim 2 \mathrm{~h}$, with array configurations $\mathrm{C}, \mathrm{DnC}$ and $\mathrm{D}$ respectively, in standard continuum mode with $2 \times 50$ contiguous bands. The same calibrators (3C 48 and 0513-219) were used.

Data reduction was carried out with the Astronomical Image Processing System (AIPS) software package. The visibility data was inspected for quality both before and after the standard calibration procedures, and noisy points were removed. For imaging the data we used the task IMAGR. We also CLEANed the region around each source and used the UVSUB routine to subtract the resulting source models for the background sources from the visibility data. For examining the light curves we used the AIPS task DFTPL.

1 The National Radio Astronomy Observatory is a facility of the National Science Foundation operated under cooperative agreement by Associated Universities, Inc.
Table 1. A summary of $I_{\mathrm{C}}$ band observations of 2MASS J052338221403022 in December 2004 and 2006. The penultimate column gives the rms scatter in the data, and the last column the range of rms scatter for the five brightest stars in the field of view.

\begin{tabular}{cccccc}
\hline \hline $\begin{array}{c}\text { Start time } \\
(\text { HJD 2450000+) }\end{array}$ & $\begin{array}{c}\text { Run } \\
(\mathrm{h})\end{array}$ & $\begin{array}{c}\text { Exp } \\
(\mathrm{s})\end{array}$ & $N$ & $\begin{array}{c}\sigma \\
(\mathrm{mmag})\end{array}$ & $\begin{array}{c}\sigma \\
(\mathrm{mmag})\end{array}$ \\
\hline 3363.3115 & 1.6 & 120 & 42 & 7 & $6-12$ \\
3366.2931 & 3.0 & 90 & 96 & 7 & $6-9$ \\
4088.4897 & 1.7 & 100 & 23 & 5 & $4-8$ \\
\hline
\end{tabular}

\subsection{Optical}

The optical observations were made in the $I_{C}$ band, with a CCD camera attached to the $1.9 \mathrm{~m}$ telescope of the South African Astronomical Observatory. The $1024 \times 1024$ CCD SITe chip gives a camera field of view of $25 \operatorname{arcmin}^{2}$ on the telescope, which allowed measurement of four bright comparison stars. The camera was operated in $2 \times 2$ prebinning mode throughout. Calibration flat-fields were obtained during evening twilight, under perfect photometric conditions.

The photometric reductions were performed with a modified version of DOPHOT (Schechter et al. 1993). The profile-fitted magnitudes were considerably more accurate than the aperture magnitudes, and only the former were retained.

An observing log is given in Table 1: the first two sets of measurements were published in Koen (2005), where time series plots of those data can be seen.

\section{Results}

2M0523, first discovered by Cruz et al. (2003) is a L2.5 brown dwarf located at a distance of 13.4 pc. It was previously reported as radio active by Berger (2006), with a flux of $231 \mu \mathrm{Jy}$ (see Fig. 1). Thus we expected that imaging the visibilities of our data would reveal a source at the position of the dwarf. To our surprise, no source was present at that position. Figure 2a shows the image of the field in total intensity (Stokes I), with the background sources present. The rms of the map is $\approx 15 \mu \mathrm{Jy}$, which gives a $3 \sigma$ upper limit of $45 \mu \mathrm{Jy}$.

Our re-analysis of the 2004 archive data sets revealed an interesting picture. There is no detection on May 3, with a $3 \sigma$ upper limit of $42 \mu \mathrm{Jy}$. We did detect the source on May 17, with a flux of $95 \mu \mathrm{Jy}$. Imaging the June 18 data revealed a yet stronger source with a flux of $230 \mu \mathrm{Jy}$ (Fig. 2b). The rms values for the May 17 and June 18 maps are $19 \mu \mathrm{Jy}$ and $17 \mu \mathrm{Jy}$ respectively.

Due to differences in the setup, the resolution of the datasets was different. In Fig. 2a, the lower resolution of the image was done by: 1) retrieving the dimensions of the CLEAN restoring beam from the header of the low resolution image (i.e. the 2004 data) and 2) specifying those dimensions in the task IMAGR when imaging the high resolution image. The same background sources are clearly visible in both maps with the absence of a source at, or even near the position of $2 \mathrm{M} 0523$ in the 2006 September 23 data. In order to check whether the 2004 May 17 or June 18 detection is dominated by a large flare, we also looked at the total intensity light-curves versus time. No flaring activity was detected.

In the optical, the present object was monitored for variability in December 2004 and 2006 (see Table 1). Although there is no evidence for optical brightness variations on timescales shorter than 2-3 h, the question of changes on timescales of days remains open. In order to investigate this, we use measurements for the five brightest stars $\left(16.4 \leq I_{\mathrm{C}} \leq 17.1\right.$, assuming 

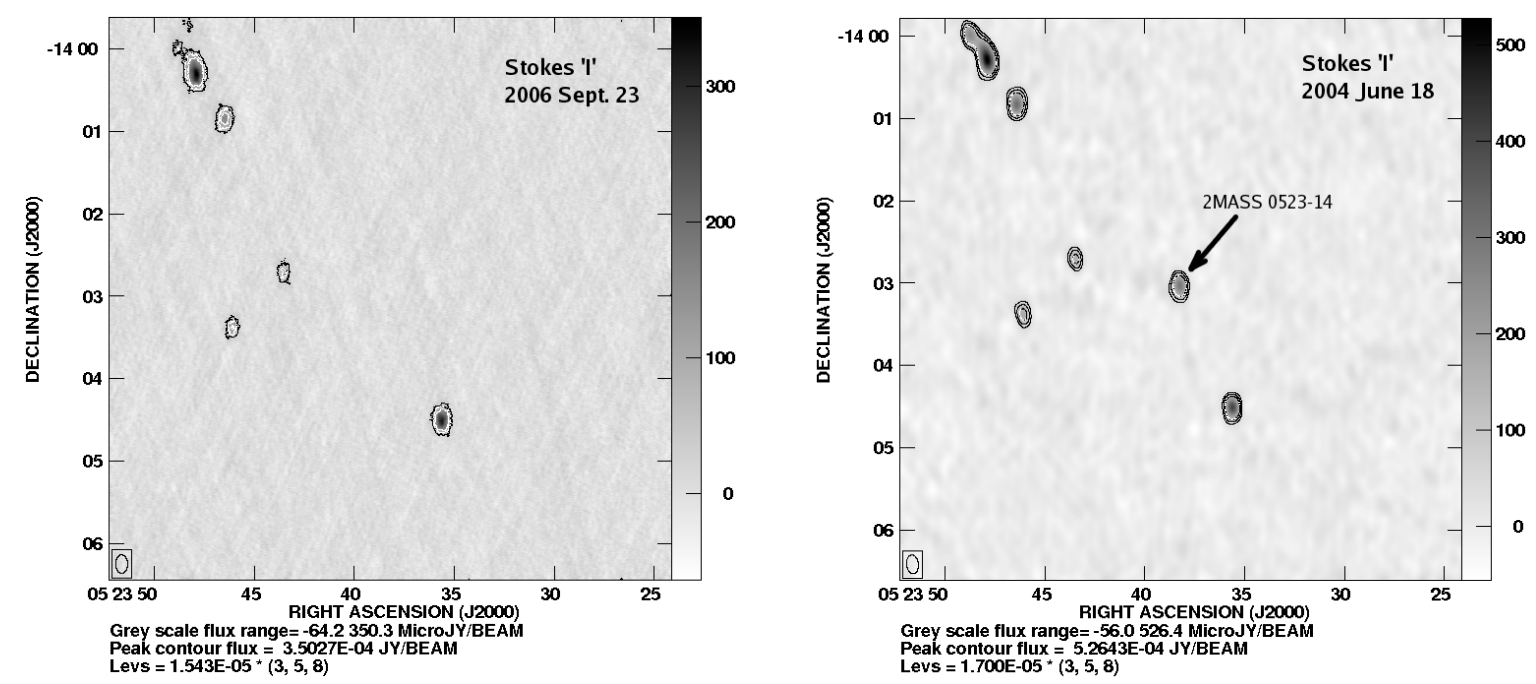

Fig. 2. VLA map of the 2MASS J05233822-1403022 field at $8.46 \mathrm{GHz}$ in total intensity (Stokes I), on 23 September 2006 and (left) on 18 June 2004 (right). The 2006 data was taken in the B configuration while the June 2004 data was in the D configuration.

$I_{\mathrm{C}}=16.6$ for $2 \mathrm{M} 0523$ - see Koen 2005). Differential magnitudes of each object were determined with respect to the means of the five stars, for each time-point observation. This gave the rms values quoted in the Table. The formal standard errors in the nightly mean magnitudes for the stars are very small - the largest is about 3.5 mmag (see Table 1). This does not, of course, take account of systematic errors, which unfortunately dominate. The night-to-night changes for the target object are larger, but only marginally. We conclude that there is currently no evidence for optical variability in $2 \mathrm{M} 0523$.

\section{Discussion}

For earlier type M dwarfs, both the quiescent and flaring coronal radio emission are generally attributed to gyrosynchrotron emission from a non-thermal population of mildly relativistic electrons with a power-law distribution. Such emission is associated with low levels of polarization and implies magnetic field strengths of up to several hundred Gauss, electron densities of $\$ 2 \times 10^{9} \mathrm{~cm}^{-3}$ and brightness temperatures of $10^{8}-10^{9} \mathrm{~K}$.

There are however some mid- $\mathrm{M}$ dwarfs whose radio emission characteristics are not well explained by the gyrosynchrotron mechanism. UV Ceti, a dM5.5e star, is one of these and has been observed to emit bright, $100 \%$ polarizied flares (Kellett et al. 2002). Similarly, AD Leo, a dM3.5e star, has shown a series of high, frequency highly polarized radio pulses during a flare (Bastian et al. 1990). Also, Burgasser \& Putman (2005), although noting that the quiescent radio emission from two ultracool dwarfs was consistent with optically thin gyrosynchrotron emission, suggested that two detected flares implied a coherent emission process. These had high brightness temperature $\left(10^{13} \mathrm{~K}\right)$, were of short duration $(\approx 4-5 \mathrm{~min})$ and were highly circular polarizied $(\approx 100 \%)$.

The characteristics of many of these bursts are consistent with the electron cyclotron maser process. For the maser operation, a population inversion in the electron distribution is needed, as well as a relatively strong magnetic field and low-density plasma, so that the electron-cyclotron frequency $v_{\mathrm{c}}$ is greater than the plasma frequency $v_{\mathrm{p}}$.

As to the instability at the heart of the process, different distributions has been proposed, e.g. Wu \& Lee (1979) suggested a loss-cone for the terrestrial auroral kilometric emission while
Pritchett (1984) suggested a shell distribution. It has since been shown that the shell instability is much more efficient than the loss-cone instability as a source of AKR (see Treumann 2006, for a review). It is suggested that the main source for any strong electron-cyclotron maser is found in the presence of a magneticfield-aligned electric potential drop which has several effects. For example, it can dilute the local plasma to such an extent that the plasma enters the regime in which the electron-cyclotron maser becomes effective and favors emission in a direction roughly perpendicular to the ambient magnetic field. This emission is the most intense, since it implies the coherent resonant contribution of a maximum number of electrons in the distribution function. What is more, such an instability can be sustained over a range of heights above the stellar surface, thus producing pseudo broadband, coherent radio emission, which would explain the simultaneous detection of both 8.4 and $4.9 \mathrm{GHz}$ emission from TVLM 513-46546 (Hallinan et al. 2006, 2007) and 2MASS0036+18 (Berger et al. 2002, 2005). This would imply kilogauss fields on ultra-cool dwarfs, which has been recently confirmed by Reiners \& Basri (2007).

Whether such a model applies in this instance is unclear since we do not have pulsed emission. There is no evidence which can conclusively distinguish between the maser and the gyrosynchrotron processes as the source of the "quiescent" radio emission detected from any UCD thus far. Assuming the persistent emission is isotropic, gyrosynchrotron radiation from an extended corona (as suggested for quiescent radio emission from early dMe stars) would imply magnetic fields up to a few hundred Gauss, brightness temperatures limited to a few times $10^{9} \mathrm{~K}$ and a source region of $\sim 2-3$ times the radius of the dwarf. The presence of such emission requires a constant supply of electrons accelerated to coronal energies. The most commonly prescribed mechanism for such acceleration is magnetic reconnection leading to micro-flaring. Yet in the cool, dense atmospheres of UCDs the magnetic field is largely decoupled from the atmosphere (Mohanty et al. 2002), and as a result the generation and propagation of magnetic stresses which can lead to magnetic reconnection is inhibited at the dwarf's surface.

However, if we assume that gyrosynchrotron is possible and since it is roughly proportional to the injection rate of electrons (Güdel 2002), thus if by some process the injection rate is reduced (for example a reduced level of micro-flaring), this would 
mean a reduced level of radio emission. In the case of 2MASS 0523 there was no evidence for flare activity during the enhanced flux levels detected in June 2004, suggesting instead a steady rise in the overall flux levels over a period of at least five weeks. Nevertheless, the "quiescent" emission from this source displays a ten-fold variation in the flux levels, which is similar to what is observed for the pulsed coherent emission from TVLM 513 (Hallinan et al. 2007). Therefore an alternative explanation for this large scale variability may be given in terms of a large dipolar-field and the electron-cyclotron maser (such emission would require magnetic field strengths of the order of several $\mathrm{kG}$, brightness temperatures $\gtrsim 10^{11} \mathrm{~K}$ and source region of up to a few tenths of the stellar radius). For such a field configuration, the plasma density would not be homogeneous throughout the magnetosphere. At present neither mechanism can be ruled out, yet it is notable that the large variations in flux with time are similar to what is observed for the pulsed emission from UCDs.

Hallinan et al. (2006) showed that TVLM 513-46546 has a well defined spot structure. The present $I$-band data suggest a lack of large-scale spots on 2M0523 in December '04 and '06, although whether spots were present during the VLA observations of June ' 04 in unclear. The possible lack of spots detected from $2 \mathrm{M} 0253$ can be explained by a series of factors. For example, $2 \mathrm{M} 0253$ is of later spectral type and hence the spots would have a lower contrast to the photosphere therefore reducing the chances of detection. It should also be noted that very high latitude spots combined with a low inclination could cause very low amplitude rotational modulation which may not be measurable. Furthermore, truly polar spots do not cause rotational modulation of the stellar brightness at all.

Such spot activity and hence the presence of increased magnetic reconnection in the lower atmosphere could lead to higher plasma levels in the outer atmosphere. Such activity could disrupt the stable electric fields in the magnetosphere of the ultra-cool dwarf. While the plasma population in the magnetosphere may re-stabilize after such an injection of plasma, the maser emission would probably switch off. The present observations therefore suggests that ultra-cool dwarfs may not just be pulsing but may also be sporadic in their emission levels, which leads to the question of how many of these ultra-cool dwarfs are radio active. For example, Berger (2006) suggested $\approx 10 \%$. However, the results presented here show that this value is dependent on the assumption that these objects are stable emitting sources. Long-term multi-frequency monitoring of the behaviour of a small sample of these objects should be undertaken before further large-scale surveys commence, in addition to further simultaneous optical and radio monitoring in order to assert the nature of activity in these objects.

However, the above interpretation of the "quiescent" radio emission (although one may question the use of the term quiescent) comes with the obvious health warning that more data is needed before we would be in a position to identify with certainty the mechanism of the "quiescent" radio emission in UCDs.

Acknowledgements. Armagh Observatory is grant-aided by the N. Ireland Dept. of Culture, Arts \& Leisure. The authors thank Stephen Bourke for his help with IRAF and Kathlin Olah for advice on spot modelling. We also gratefully acknowledge the support of the HEA funded Cosmogrid project and Enterprise Ireland under the grant award SC/2001/0322, plus PPARC for a visitor grant.

\section{References}

Bastian, T. S., Bookbinder, J., Dulk, G. A., \& Davis, M. 1990, ApJ, 353, 265 Benz, A. O., Conway, J., \& Güdel, M. 1998, A\&A, 331, 596

Berger, E. 2002, ApJ, 572, 503

Berger, E. 2006, ApJ, 648, 629

Berger, E., Ball, S., Becker, K. M., et al. 2001, Nature, 410, 338

Berger, E., Rutledge, R. E., Reid, I. N., et al. 2005, ApJ, 627, 960

Burgasser, A. J., \& Putman, M. E. 2005, ApJ, 626, 486

Cruz, K. L., Reid, I. N., Liebert, J., Kirkpatrick, J. D., \& Lowrance, P. J. 2003, AJ, 126, 2421

Güdel, M. 2002, ARA\&A, 40, 217

Güdel, M., \& Benz, A. O. 1993, ApJ, 405, L63

Hallinan, G., Antonova, A., Doyle, J. G., et al. 2006, ApJ, 653, 690

Hallinan, G., Lane, C., Bourke, S., et al. 2007, ApJL, submitted

Kellett, B. J., Bingham, R., Cairns, R. A., \& Tsikoudi, V. 2002, MNRAS, 329, 102

Koen, C. 2005, MNRAS, 360, 1132

Mohanty, S., Basri, G., Shu, F., Allard, F., \& Chabeier, G. 2002, ApJ, 571, 469

Osten, R. A., Hawley, S. L., Bastian, T. S., \& Reid, I. N. 2006, ApJ, 637, 518

Pritchett, P. L. 1985, Geophys. Res., 89, 8957

Reiners, A., \& Basri, G. 2007, ApJ, 656, 1121

Schechter, P. L., Mateo, M., \& Saha, A. 1993, PASP, 105, 1342

Schmidt, S. J., Cruz, K. L., Bongiorno, B. J., Liebert, J., \& Reid, I. N. 2007 [arXiv: astro-ph/0701055]

Treumann, R. A. 2006, A\&AR, 13, 229

Phan-Bao, N., Osten, R. A., Lim, J., Martin, E. L., \& Ho, P. T. P. 2007, ApJ, 658, 553

Wu, C. S., \& Lee, L. C. 1979, ApJ, 230, 621 